\title{
Editorials
}

\section{Anesthesiology and hyperbaric medicine}

Kenneth M. LeDez MB ChB, FRCPC

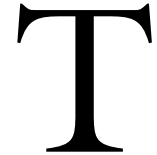

HE paper by Dr. Wherrett and colleagues in this issue ${ }^{1}$ underlines the necessity of instituting hyperbaric oxygen treatment for suspected iatrogenic arterial gas embolism even if a delay of days has occurred. The commonest reasons for delay are a failure to consider the diagnosis and treatment; concern related to the critical status of the patient; and a mistaken belief that the patient is not fit for transportation or treatment in a hyperbaric chamber. Hospital-based chambers are capable of invasive monitoring and treatment, including drug infusions and mechanical ventilation. Transportation to a hyperbaric treatment centre by road, or by air at low altitude (less than 1,000 feet), or preferably in an aircraft pressurized to sea level, ${ }^{2}$ is virtually always possible within a matter of hours. The associated costs of transportation and operation of hyperbaric treatment facilities are small in comparison to the costs of major neurological damage.

Numerous (in fact hundreds of) cases of cerebral arterial gas embolism have been witnessed, especially during military training for submarine escape ${ }^{3}$ but also during a variety of medical procedures. Hyperbaric chambers are located at the top of navy submarine escape training tow ers for this reason. The often-speedy effectiveness of hyperbaric oxygen treatment has been witnessed so many times it is beyond dispute. Hyperbaric oxygen is the definitive treatment. One recent unpublished case in St. John's, Newfoundland, was due to disconnection of a central venous pressure line and again was effectively treated with hyperbaric oxygen.

A skeptic could argue that an increased sedation requirement does not constitute conclusive evidence of the efficacy of hyperbaric oxygen in the case described by Dr. Wherrett. However, a combative patient inside the confines of a hyperbaric chamber is a frightening experience indeed, particularly once the decompression obligation of staff prohibits depressur- ization. This has led to a trend towards greater use of $i v$ anesthesia and sedation particularly for patients requiring positive pressure ventilation inside hyperbaric chambers. The case described highlights the close link between anesthesia and hyperbaric medicine. No doubt this link contributed to the implementation, albeit delayed, of the only treatment known to be specific and effective in arterial gas embolism and the consequent favourable outcome.

Apart from administration of anesthesia during hyperbaric treatment, expertise in monitoring, ventilation, breathing systems, and critical care link the two areas of practice together. Anesthesiology and hyperbaric medicine also share an interest in physics, the gas laws, compressed gases, pharmacology, and physiology. Like anesthesia, remuneration in hyperbaric medicine is generally based upon time periods.

Hyperbaric medicine is a small area of clinical practice in Canada that is without a separate Canadian journal, society, or certification mechanism. Many hyperbaric centres are closely associated with departments of anesthesia. Facilities in St. John's, Toronto, Ottawa, Hamilton, and Edmonton were established by or with assistance from anesthesiologists. Hospitalbased hyperbaric facilities also exist in Halifax, Montreal and Vancouver. A similar situation is found in countries such as the USA, UK, Australia, and Russia. Strong links have also developed between these facilities and intensive care units.

While anesthesiologists are perhaps the largest single specialist group involved in hyperbaric medicine, others include ex-navy physicians, family physicians, respirologists, neurologists, intensivists, and surgeons. A number of anesthesiologists have undertaken hyperbaric medicine fellowships, and recently an anesthesia resident undertook an elective rotation in hyperbaric medicine in St. John's. Future certification may necessitate the small field of hyperbaric medicine becoming 
formally associated with another, perhaps to the specialty of anesthesiology.

Like many other industrialized countries Canada depends upon occupational divers for numerous underwater construction and maintenance tasks (e.g., maintenance of water supply intakes, repair and construction of wharves, ship and boat repairs, etc.). Recreational diving is increasingly popular. Decompression illnesses are important indications for emergency hyperbaric oxygen treatment. Other emergency conditions such as crush injuries also benefit. ${ }^{4}$ The most common elective clinical uses are for tissue damage following cancer irradiation, for non-healing problem wounds, failing skin grafts and flaps. These uses are supported by clinical trials and extensive reviews. ${ }^{5-9}$

There are different types of hyperbaric chambers, from monoplace units capable of pressures of about 2.5 atmospheres up to the large complex chambers used for deep offshore diving. The principle non-capital costs are for staff and maintenance. An emergency treatment generally requires from three to six staff, depending upon the patient and the type of chamber, and this is comparable to the hourly cost of an operating room. Since months of hospital care and rehabilitation would undoubtedly cost much more and produce a worse outcome the cost effectiveness of hyperbaric oxygen in a case like the one described is obvious.

Hyperbaric oxygen has been used inappropriately for many conditions in the past. Health Canada recently issued a directive that hyperbaric chambers should not be used in the treatment of neurological conditions such as cerebral palsy and stroke except as part of an ethically conducted trial. ${ }^{10}$ The Undersea and Hyperbaric Medical Society periodically reviews the scientific evidence for the use of hyperbaric oxygen and publishes a list of "approved" conditions. ${ }^{11}$ All Canadian provincial health care plans pay for hyperbaric treatments of these approved conditions. Private facilities treating non-approved conditions continue to operate in defiance of Health Canada Directives and make outrageous claims of benefit. Hospital-based facilities treating expertly selected cases have a good record of cost-effective successes and, as in this case, anesthesiologists are often involved.

\section{Anesthésiologie et médecine hyperbare}

L'article du Dr Wherrett et de ses collègues souligne, dans le présent numéro, ${ }^{1}$ la nécessité d'instaurer un traitement à l'oxygène hyperbare pour l'embolie gazeuse artérielle iatrogénique présumée, même après un délai de quelques jours. Les raisons habituelles d'un retard sont un défaut d'avoir envisagé le diagnostic et le traitement, une préoccupation liée à l'état critique du patient et une croyance erronée que le patient ne pourra supporter le transport ou le traitement en caisson hyperbare. Les caissons en milieu hospitalier permettent un monitorage et un traitement effractifs, y compris les perfusions de médicaments et la ventilation mécanique. Le transport vers un centre de traitement hyperbare, par la route ou la voie des airs à basse altitude (moins de 1000 pieds), ou de préférence par un avion dont la pression est réglée sur celle du niveau de la mer, ${ }^{2}$ est presque toujours une question d'heures. Le coût associé au transport et au fonctionnement des installations d'oxygénothérapie hyperbare est bas en comparaison de celui des dommages neurologiques sévères.

Nombre de cas d'embolie gazeuse artérielle cérébrale, des centaines en fait, ont été authentifiés, surtout pendant la formation militaire pour l'évacuation de sous-marins, ${ }^{3}$ mais aussi pendant diverses interventions médicales. Les caissons hyperbares sont, pour cette raison, situées en haut des tours d'entraînement utilisées pour la formation à l'évacuation des sousmarins. L'efficacité souvent rapide de l'oxygénothérapie hyperbare a été attestée maintes et maintes fois, on ne saurait donc la contester. C'est un traitement définitif. Un récent cas non publié, survenu à St-Jean de TerreNeuve, a été causé par la déconnexion d'une sonde de pression veineuse centrale et, encore une fois, traité efficacement avec de l'oxygène hyperbare.

Les opposants au traitement pourraient soutenir que les besoins accrus de sédation ne constituent pas une preuve concluante de l'efficacité de l'oxygène hyperbare dans le cas décrit par le Dr Wherrett. Cependant, la présence d'un patient agité dans l'espace restreint du caisson hyperbare représente une expérience angoissante réelle, en particulier parce que l'obligation de décompression du personnel interdit la dépressurisation. Cette situation a évolué vers un plus grand usage d'anesthésie et d'analgésie iv surtout pour les patients nécessitant une ventilation à pression 
positive à l'intérieur du caisson. Le cas décrit met en relief le lien étroit entre l'anesthésie et la médecine hyperbare. Il ne fait aucun doute que ce lien a contribué à l'implantation, quoique retardée, du seul traitement reconnu comme spécifique et efficace de l'embolie gazeuse artérielle et à l'évolution favorable qui en résulte.

Indépendamment de l'administration de l'anesthésie pendant le traitement hyperbare, l'expertise en monitorage, en ventilation, en systèmes respiratoires et en réanimation rassemble les deux domaines de pratique. L'anesthésiologie et la médecine hyperbare partagent aussi un intérêt pour la physique, les lois des gaz, les gaz comprimés, la pharmacologie et la physiologie. Comme en anesthésie, la rémunération en médecine hyperbare est généralement fondée sur les tranches horaires.

La médecine hyperbare n'occupe qu'un champ restreint de pratique au Canada, sans journal, association professionnelle ou mécanisme d'accréditation distincts. De nombreux centres hyperbares sont étroitement associés à des services d'anesthésie. Les installations de StJean, Toronto, Ottawa, Hamilton et Edmonton ont été réalisées par, ou avec l'assistance, des anesthésiologistes. Les aménagements hyperbares en milieu hospitalier existent également à Halifax, Montréal et Vancouver. On retrouve une situation similaire aux États-Unis, au Royaume-Uni, en Australie et en Russie. Des liens solides se sont aussi développées entre ces centres et les unités de soins intensifs.

Les anesthésiologistes sont sans doute le seul groupe important de spécialistes impliqués en médecine hyperbare, mais on compte aussi d'anciens médecins de la marine, des médecins de famille, des spécialistes des troubles respiratoires, des neurologues, des intensivistes et des chirurgiens. Nombre d'anesthésiologistes ont entrepris des recherches en médecine hyperbare et, récemment, un résident en anesthésie à St-Jean a choisi une rotation facultative dans ce domaine. Une future accréditation pourrait nécessiter la réunion formelle du petit groupe de médecine hyperbare avec une autre association, peutêtre celle des anesthésiologistes.

À l'instar de nombreux autres pays industrialisés, le Canada compte sur des plongeurs professionnels pour beaucoup de tâches de construction et d'entretien subaquatiques (par ex., l'entretien des conduites d'approvisionnement d'eau, la réparation et la construction des quais, la réparation des navires et des bateaux, etc.) . La plongée est un loisir de plus en plus populaire. Les maux de décompression sont d'importantes indications pour un traitement urgent à l'oxygène hyperbare. D'autres conditions d'urgence, comme les syndromes de traumatismes par écrasement en bénéfi- cient également. ${ }^{4}$ L'usage clinique non urgent le plus fréquent concerne les lésions tissulaires consécutives à la radiothérapie pour le cancer, les problèmes de cicatrisation, l'échec des greffes et des lambeaux cutanés. Ces usages sont confirmés par des essais cliniques et d'importantes revues de littérature. ${ }^{5-9}$

Il existe différents types de caissons hyperbares, allant des unités monoplaces capables de pressions d'environ 2,5 atmosphères jusqu'aux grandes chambres complexes utilisées pour la plongée en eau profonde. Le principal coût de fonctionnement concerne le personnel et l'entretien. Un traitement d'urgence demande généralement de trois à six membres du personnel, selon le patient et le type de caisson. Le coût horaire est comparable à celui d'une salle d'opération. Comme des mois d'hospitalisation et de réadaptation coûteraient sûrement beaucoup plus et ne donneraient pas de meilleurs résultats, la rentabilité de l'oxygène hyperbare dans un cas comme celui qu'on a décrit est évidente.

L'oxygène hyperbare a été utilisé de façon inappropriée pour de nombreuses maladies dans le passé. Santé Canada a émis récemment une directive disant que les caissons hyperbares ne devraient pas être utilisés dans le traitement des troubles neurologiques comme la paralysie cérébrale et les accidents vasculaires, sauf si cela fait partie d'un essai accepté par un comité d'éthique. ${ }^{0}$ La Undersea and Hyperbaric Medical Society réexamine périodiquement la preuve scientifique de l'usage de l'oxygène hyperbare et publie une liste des maladies "approuvées." 1 Tous les plans de santé provinciaux canadiens paient les traitements hyperbares de ces maladies. Les centres privés qui offrent des traitements non approuvés continuent de fonctionner en défiant les Directives de Santé Canada et présentent de scandaleuses prétentions de bienfaits. Les installations hospitalières qui offrent le traitement de cas choisis ont un bilan positif et rentable et, comme dans le cas présenté, les anesthésiologistes y participent souvent.

\section{References}

1 Wherrett CG, Mehran RJ, Beaulieu M-A Cerebral arterial gas embolism following diagnostic bronchoscopy: delayed treatment with hyperbaric oxygen. Can J Anesth 2002; 49: 96-99.

2 Bennett PB, Elliott DH. The Physiology and Medicine of Diving, $4^{\text {th }}$ ed. London: W.B Saunders Company Ltd, 1993.

3 Edmonds, Lowry, Pennefather. Diving and Subaquatic Medicine, $3^{\text {rd }}$ ed. Butterworth-Heinemann Ltd., 1992.

4 Bouachour G, Cronier P, Gouello JP, Toulemonde JL, Talha A, Alquier $P$. The Journal of trauma: injury, infection, and critical care 1996; 41; 333-9. 
5 Marx RE, Johnson RP, Kilne SN. Prevention of osteoradionecrosis: a randomized prospective clinical trial of hyperbaric oxygen versus penicillin. JADA 1985; 111 : 49-54.

6 Perrins DJ. Influence of hyperbaric oxygen on the survival of split skin grafts. Lancet 1967; 7495: 868-71.

7 Hammarlund C, Sundberg T. Hyperbaric oxygen reduced size of chronic leg ulcers: A randomized double- blind study. Plast Reconstr Surg 1994; 93: 829-33.

8 Feldmeier JJ, Heimbach RD, Davolt DA, et al. Hyperbaric oxygen as an adjunctive treatment for severe laryngeal necrosis: a report of nine consecutive cases. Undersea Hyperb Med 1993; 20: 329-35.

9 Bevers RFM, Bakker DJ, Kurth KH. Hyperbaric oxygen treatment for haemorrhagic radiation cystitis. Lancet 1995; 346: 803-5.

10 Associations from the Therapeutic Products Program of Health Canada. Hyperbaric Oxygen Chambers (Letter). (Note: available on the Health Canada website), June 16, 2000.

11 Hyperbaric Oxygen Therapy Committee Report 1999. Undersea and Hyperbaric Medical Society, Bethesda, Maryland, USA, 1999. 